\title{
Effect of Heat Preconditioning on Ultrastructural Alterations and S-100 Immunoreactivity in the Dentate Gyrus of the Heatstroke-Induced Rat
}

\author{
S.M. Yu, ${ }^{*}$ W.H. Han, ${ }^{* *}$, and K.H. Lin*** \\ * Department of Nursing, Central Taiwan University of Science and Technology, \\ Taichung 406, Taiwan \\ ** Department of Anatomy and Cell Biology, National Yang-Ming University, Taipei \\ 112, Taiwan \\ *** Graduate Institute of Physical Therapy, School of Physical Therapy, National \\ Taiwan University, Taipei 100, Taiwan
}

Heatstroke-induced neuronal damage can result from the heat and lower perfusion of cerebral blood flow [1]. We investigated the morphological changes of neurons and astroglia in the CA3 and CA4 sectors of hippocampus and dentate gyrus in heatstroke-induced rats by using immunocytochemistry, electron microscopy and Western blotting. Sprague-Dawley rats were pre-treated in a $45^{\circ} \mathrm{C}$ heatbox for 30 min. On the following day after one-day recovery, the animal was incubated in a heatbox to induce the formation of heatstroke. At $30 \mathrm{~min}$ after heatstroke formation, the histological morphology and fine structure were observed. At light microscopic level, S-100-like immunoreacrive positive cells increased moderately in their nuclei and cytoplasmic processes. Such cells were abutted on the blood vessel and associated with perikaryonic dilation. At the electron microscopic level, the edematous dendrite was close to the healthy-looking neuron in the granule layer of the dentate gyrus (Fig. 1A). The perikaryotic dilation was observed in the astrocyte of the subgranular layer (Fig. 1B). The vacuole of the astrocyte was also located in the perivascular end feet abutted on the blood vessel (Fig. 1C). The edematous process of the astrocyte was located lying near the electron-dense neuron in the molecular layer of the dentate gyrus (Fig. 1D).

[1] M.T. LIn, Clin. Exp. Pharmocol. Physiol.26 (1999) 826. 

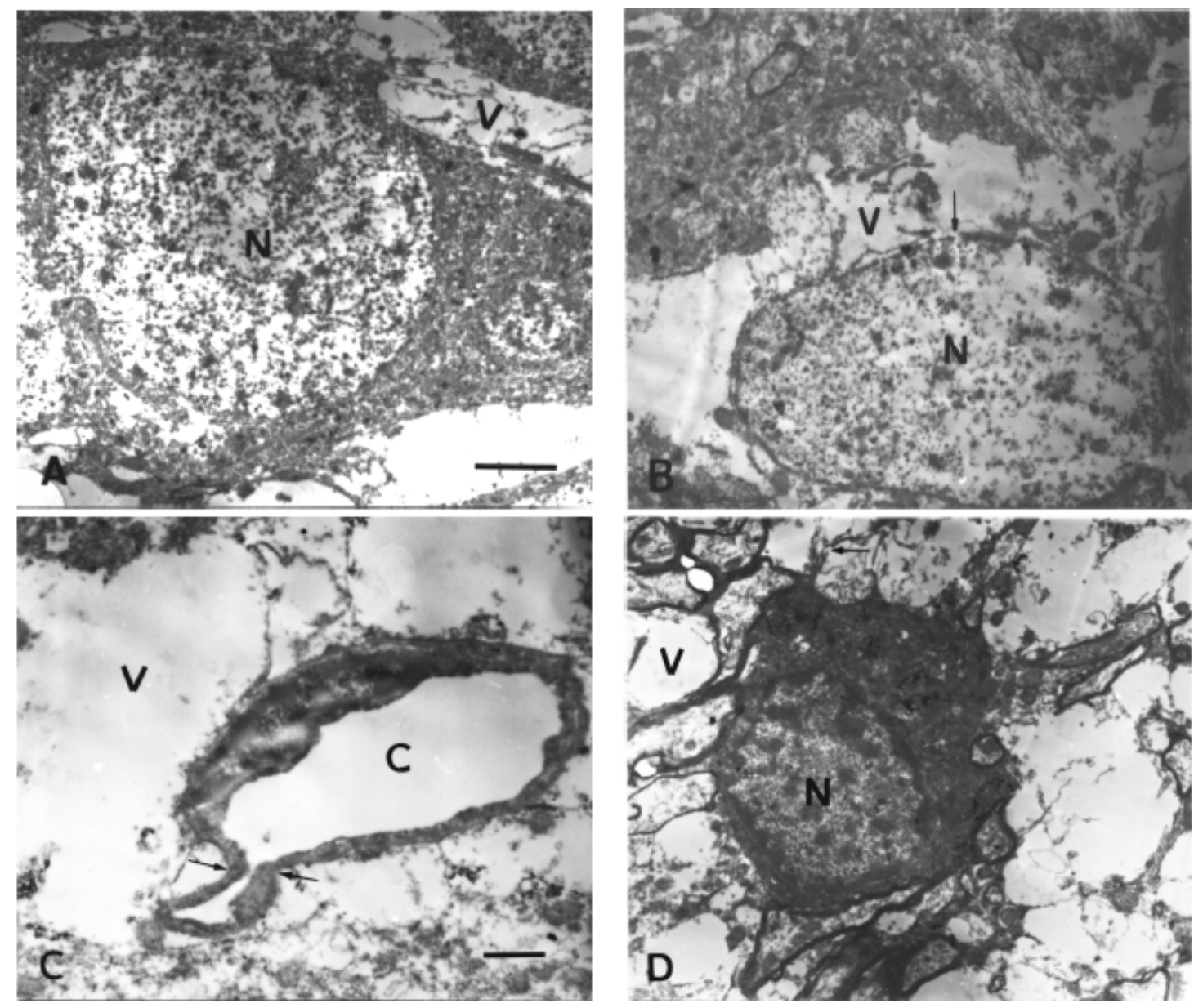

FIG. 1. Electron micrographs of the granule layer in the dentate gyrus at $30 \mathrm{~min}$ after the heatstroke following heat preconditioning. A. A healty-looking neuron $(\mathrm{N})$ in the granule layer of the dentate gyrus. Note the edematous dendrite (V). B. An astrocyte $(\mathrm{N})$ with perikaryonic dilation (V) and incomplete nuclear membrane (arrow). C. The vacuole (V) of the astrocyte abutted on the blood vessel (C). D. The vacuole $(\mathrm{V})$ of the astrocyte lying near the electron-dense neuron $(\mathrm{N})$. Scale Bar: $2 \mu \mathrm{m}(\mathrm{A}-\mathrm{B}, \mathrm{C}-\mathrm{D})$. 